\title{
Rational Transform Approximation via the Laguerre Spectrum
}

by KENNETH STEIGLITZ

Department of Electrical Engineering
Princeton University, Princeton, New Jersey

ABSTRACT: A method is presented for determination of an $n^{\text {th }}$ order rational transform approximation for a time function, given at least $n+1$ of its Laguerre coefficients. The method is based on approximating the discrete set of Laguerre coefficients with a rational generating function. The method does not require predetermination of the poles; and allows the use of as many Laguerre coefficients as are available, without increasing the complexity of the model. Applications to time domain synthesis and transfer function identification are discussed.

\section{Introduction}

An important class of functions from the point of view of signal representation and approximation is the class with $n^{\text {th }}$ order rational Laplace transforms. These functions arise naturally in such fields as model identification and timedomain synthesis. The problem of finding the best $n^{\text {th }}$ order rational function to represent a given time function is, therefore, important; unfortunately it is beset with difficulties. If a truncated orthonormal expansion is used, the pole locations must be predetermined by some a priori means, and the order is determined by the number of orthonormal functions used $(1,2)$. Fourier series techniques involve compounded approximations and do not afford a clear picture of the errors involved $(3,4)$. Sampled-data techniques involve aliasing problems which also make errors difficult to evaluate (5-10). None of these methods clearly shows how close the result is to the best (in some prescribed sense) $n^{\text {th }}$ order rational function.

In this paper an approach is presented which uses the generating function for the Laguerre coefficients of an arbitrary time function. This generating function is shown to be proper and rational with $n$ poles if and only if the time function has a Laplace transform which is proper and rational with $n$ poles. The problem of signal representation and approximation is transformed, therefore, into one of finding good rational generating functions for a discrete series of coefficients-which is, in many ways, a more tractable problem than the original.

This approach is advantageous in that the poles are not preassigned and that an arbitrarily large number of Laguerre coefficients can be used to find a low order transform. Because of the norm-preserving character of the transformation introduced, the error analysis is especially clear. Three types of errors contribute to the overall representation or approximation error: 


\section{Kenneth Steiglitz}

1) The errors in measuring or calculating the Laguerre coefficients of a signal or impulse response;

2) The error caused by using only a finite number of these coefficients;

3) The error in fitting the rational generating function to the given coefficients.

These errors, all more or less under the control of the user, can often be easily estimated. The root-integral-square-error of the final result is bounded by the sum of these three root-integral-square-errors.

A method based on regression techniques is presented for fitting a generating function to the Laguerre coefficients. This method is not optimal but is easy to use and has proven dependable and practical in many situations. An example of an application to lumped delay-line synthesis is discussed.

\section{Representation of Functions with $\boldsymbol{n}^{\text {th }}$ Order Rational Transforms}

A particularly important set of complete orthonormal functions on $(0, \infty)$ are the Laguerre functions, discussed by Wiener (11),

$$
\lambda_{n}(t)=\mathfrak{L}^{-1}\left[\frac{\sqrt{2}}{1+s}\left(\frac{1-s}{1+s}\right)^{n}\right], \quad n=0,1,2, \cdots
$$

Any function in $L^{2}(0, \infty)$ can be expressed as a limit-in-the-mean of a sum of these functions

$$
f(t)=\sum_{n=0}^{\infty} f_{n} \lambda_{n}(t)
$$

where

$$
f_{n}=\int_{0}^{\infty} f(t) \lambda_{n}(t) d t
$$

are the Laguerre coefficients of $f(t)$ and comprise its Laguerre spectrum. We define the Laguerre transform of $f(t)$ as the generating function of its Laguerre spectrum as follows:

Definition I: Let $f(t) \in L^{2}(0, \infty)$. The function of $z$

$$
\mathbf{F}(z)=\sum_{n=0}^{\infty} f_{n} z^{-n}
$$

where $f_{n}$ is given by Eq. 1, is termed the Laguerre transform of $f(t)$. When $z=\exp (j \omega)$, this function exists as a limit-in-the-mean and is in $L^{2}(0,2 \pi)$.

The following is a simple relationship between $F(s)=F(j \omega)$, the Fourier transform of $f(t)$, and $F(z)$.

\section{Theorem I:}

$$
\mathbf{F}(z)=\frac{\sqrt{2} z}{z+1} F\left(\frac{z-1}{z+1}\right)
$$


Proof: An application of the $F$. Riesz-Fischer theorem (12) yields

$$
f_{n}=\frac{1}{2 \pi j} \oint \mathbf{F}(z) z^{n} \frac{d z}{z} \quad n=0,1,2, \cdots,
$$

where the integration is carried out around the unit circle. Letting $z=\frac{1+s}{1-s}$ in this, gives

where

$$
f_{n}=\frac{1}{2 \pi j} \int_{-j \infty}^{j \infty} \frac{\sqrt{2}}{1+s} \mathbf{F}\left(\frac{1+s}{1-s}\right) \Lambda_{n}(-s) d s
$$

$$
\Lambda_{n}(s)=\frac{\sqrt{2}}{1+s}\left(\frac{1-s}{1+s}\right)^{n} \quad n=0,1,2, \cdots
$$

which is the Laplace transform of $\lambda_{n}(t)$. Hence, by Parseval's theorem,

and

$$
F(s)=\frac{\sqrt{2}}{1+s} \mathbf{F}\left(\frac{1+s}{1-s}\right)
$$

$$
\mathbf{F}(z)=\frac{\sqrt{2} z}{z+1} F\left(\frac{z-1}{z+1}\right) .
$$

It also follows from Parseval's relation that

$$
\int_{0}^{\infty}|f|^{2} d t=\sum_{n=0}^{\infty}\left|f_{n}\right|^{2}=\frac{1}{2 \pi j} \int_{-j \infty}^{j \infty}|F(s)|^{2} d s=\frac{1}{2 \pi j} \oint|\mathbf{F}(z)|^{2} \frac{d z}{z} .
$$

Theorem I gives a simple method for computing the Laguerre spectrum of a function with a rational Laplace transform. For example, consider

$$
\begin{aligned}
f(t) & =e^{-t} \sin (t) u(t) \\
F(s) & =\frac{1}{(s+1)^{2}+1} \\
\mathbf{F}(z) & =\frac{\sqrt{2}(z+1) z}{5 z^{2}+2 z+1}=\sqrt{2}\left[\frac{1}{5}+\frac{3}{25} z^{-1}-\frac{11}{125} z^{-2}+\cdots\right],
\end{aligned}
$$

so that

$$
f(t)=\sqrt{2}\left[\frac{1}{5} \lambda_{0}(t)+\frac{3}{25} \lambda_{1}(t)-\frac{11}{125} \lambda_{2}(t)+\cdots\right] .
$$

It follows from Eq. 2 that functions with rational proper Laplace transforms have rational proper Laguerre transforms. For convenience, we introduce the following:

Definition II: A function of $x$ is said to be in $R_{n}(x)$ if it is of the form

$$
\frac{\sum_{i=0}^{n-1} a_{i} x^{i}}{\sum_{i=0}^{n} b_{i} x^{i}} .
$$


Theorem II: It follows that $f(t) \in L^{2}(0, \infty)$ has a Laplace transform in $R_{n}(s)$ if and only if its Laguerre transform is in $R_{n}\left(z^{-1}\right)$. In this case $F(s)$ has all its poles in the open left-hand $s$-plane and $\mathbf{F}(z)$ has all its poles in the open unit disc.

Proof: Let $F(s) \in R_{n}(s)$. Then

$$
\mathbf{F}(z)=\frac{\sqrt{2} z}{z+1} \frac{\sum_{i=0}^{n-1} a_{i}\left(\frac{z-1}{z+1}\right)^{i}}{\sum_{i=0}^{n} b_{i}\left(\frac{z-1}{z+1}\right)^{i}} \in R_{n}\left(z^{-1}\right) .
$$

The converse follows similarly. The last remark of the theorem follows from the fact that the bilinear transformation maps the left-half plane to the unit disc. The zero of $F(s)$ at infinity maps into $z=-1$, but in virtue of the factor $\sqrt{2} z /(z+1)$ no zero of $F(z)$ necessarily occurs at $z=-1$.

\section{The Approximation Problem}

The approximation problem, discussed in the introduction, is formulated as follows: Given $f(t) \in L^{2}(0, \infty)$, find $f(t)$ such that

$$
\hat{F}(s)=\mathcal{L}[\hat{f}(t)] \in R_{n}(s) .
$$

and the integral-square-error

$$
\|f-\hat{f}\|^{2}=\int_{0}^{\infty}|f-\hat{f}|^{2} d t
$$

is small. Using the representation of the previous section, we restate the problem as follows: Given $\left\{f_{n}\right\}_{0}^{\infty}$ such that

$$
\sum_{n=0}^{\infty}\left|f_{n}\right|^{2}<\infty
$$

find a sequence $\left\{\hat{f}_{n}\right\}_{0}^{\infty}$ such that

$$
\hat{\mathbf{F}}(z)=\sum_{n=0}^{\infty} \hat{f}_{n} z^{-n} \in R_{n}\left(z^{-1}\right)
$$

and the summed-squared-error

$$
\|f-\hat{f}\|^{2}=\sum_{n=0}^{\infty}\left|f_{n}-\hat{f}_{n}\right|^{2}
$$

is small.

In a practical situation only a finite number of Laguerre coefficients can be obtained, and these inevitably are corrupted by measurement or calculation noise. Symbolize the finite observed set by

$$
f_{0}^{*}, f_{1}^{*}, \cdots, f_{N}^{*}
$$


put

and define

$$
f_{i}^{*}=0 \quad \text { for } \quad i>N
$$

$$
\mathbf{F}^{*}(z)=\sum_{i=0}^{N} f_{i}^{*} z^{-i}
$$

Assume throughout that $n \leq N$. It is now necessary to find two polynomials

and

$$
N(z)=\alpha_{0}+\alpha_{1} z^{-1}+\cdots+\alpha_{n-1} z^{-(n-1)}
$$

such that

$$
D(z)=1+\beta_{1} z^{-1}+\cdots+\beta_{n} z^{-n}
$$

$$
\frac{1}{2 \pi j} \oint\left|\frac{N(z)}{D(z)}-\mathbf{F}^{*}(z)\right|^{2} \frac{d z}{z}=\sum_{i=0}^{\infty}\left|\hat{f}_{i}-f_{i}^{*}\right|^{2}=\min
$$

Then $\hat{\mathbf{F}}(z)=\frac{N(z)}{D(z)}$ is the least-integral-square-error approximation to $\mathbf{F}^{*}(z)$, the measured or calculated version of $\mathbf{F}(z)$. Hence $\hat{F}(s)$ is the $n^{\text {th }}$ order rational function closest to $F(s)$ in $L^{2}$-norm by Eq. 3, based on the information contained in the $f_{i}{ }^{*}$. Unfortunately the minimization of Eq. 4 requires the solution of highly nonlinear regression equations for the $\beta_{i}{ }^{1}$

A closely related problem that can be solved exactly is finding $N(z)$ and $D(z)$ such that

$$
\frac{1}{2 \pi j} \oint\left|N(z)-D(z) \mathbf{F}^{*}(z)\right|^{2} \frac{d z}{z}=\min
$$

This leads to linear regression equations for the $\beta_{i}$ (14) which are easy to solve and which have been used successfully by the author for a number of problems.

The solution of Eq. 5 is derived as follows: Let

$$
D(z) \mathrm{F}^{*}(z)=\sum_{k=0}^{n+N} y_{k} z^{-k}
$$

Whatever the values chosen for the coefficients of $D(z)$, it is clear that the minimization of Eq. 5 must result in choosing

$$
N(z)=y_{0}+y_{1} z^{-1}+\cdots+y_{n-1} z^{-(n-1)},
$$

which cancels the first $n$ terms of Eq. 6 . This ensures that $N / D$ and $\mathbf{F}^{*}(z)$ agree in the first $n$ terms. Assuming that $N(z)$ is chosen in this way, Eq. 5 becomes

where

$$
\frac{1}{2 \pi j} \oint\left|N-D \mathrm{~F}^{*}\right|^{2} \frac{d z}{z}=\sum_{k=n}^{n+N} y_{k}^{2}
$$

$$
y_{k}=f_{k}^{*}+\sum_{j=1}^{n} \beta_{j} f_{k-j} \quad k=n, n+1, \cdots
$$

${ }^{1}$ An exact solution is unknown to the author, although iterative techniques appear promising. 


\section{Kenneth Steiglitz}

Differentiating Eq. 8 with respect to $\beta_{i}$ and setting the result equal to zero yields the following set of linear equations for the $\beta_{j}$ :

$$
\sum_{j=1}^{n} \beta_{j} \sum_{k=n}^{n+N} f_{k-i}^{*} f_{k-j}^{*}=-\sum_{k=n}^{n+N} f^{*}{ }_{k-i} f^{*}{ }_{k} \quad i=1, \cdots, n .
$$

Solving for the $\beta_{j}$ involves the inversion of this $n \times n$ matrix equation. After thus finding $D(\boldsymbol{z})$, there results from $\mathrm{Eq} .7$

$$
N(z)=\text { first } n \text { terms in } D(z) \mathbf{F}^{*}(z) .
$$

Using Eq. 2 the approximant becomes

$$
\hat{F}(s)=\frac{\sqrt{2}}{1+s} \frac{N\left(\frac{1+s}{1-s}\right)}{D\left(\frac{1+s}{1-s}\right)} .
$$

We have gone from an original $f(t)$ to a set of observed Laguerre coefficients $f^{*}$, to a rational generating function for these $f^{*}$, to a rational function of $s$. The error in $L^{2}$-norm is

$$
\begin{aligned}
& \left(\int_{0}^{\infty}|f-\hat{f}|^{2} d t\right)^{1 / 2} \\
& =\|f-\hat{f}\|=\|\hat{\mathbf{F}}(z)-\mathbf{F}(z)\| \leq\left\|\mathbf{F}(z)-\mathbf{F}^{*}(z)\right\|+\left\|\mathbf{F}^{*}(z)-\hat{\mathbf{F}}(z)\right\| \\
& =\left(\sum_{i=0}^{N}\left|f_{i}-f^{*}{ }_{i}\right|^{2}+\sum_{i=N+1}^{\infty}\left|f_{i}\right|^{2}\right)^{1 / 2}+\left(\sum_{i=n+1}^{\infty}\left|f^{*}{ }_{i}-\hat{f}_{i}\right|^{2}\right)^{1 / 2} \\
& \quad \leq\left(\sum_{i=0}^{N}\left|f_{i}-f^{*}{ }_{i}\right|^{2}\right)^{1 / 2}+\left(\sum_{i=N+1}^{\infty}\left|f_{i}\right|^{2}\right)^{1 / 2}+\left(\sum_{i=n+1}^{\infty}\left|f^{*}{ }_{i}-\hat{f}_{i}\right|^{2}\right)^{1 / 2},
\end{aligned}
$$

by two applications of the triangle inequality. The first term in Eq. 11 represents the measurement or calculation noise of the Laguerre coefficients; the second term represents the truncation error caused by using only a finite number of Laguerre coefficients; and the last term represents the fitting error. By the completeness of the Laguerre functions on $(0, \infty)$ the truncation error can be made arbitrarily small by choosing $N$ sufficiently large; this can be done without changing $n$, the order of the approximation. The fitting error can be computed from the given data, and the measurement or calculation error is usually known approximately. Thus an upper bound on the integral-squareerror can be estimated in a typical identification or synthesis problem.

\section{Example of Application to Time Domain Synthesis}

To illustrate the method described, the rational transform approximation of the delayed impulse

$$
f(t)=\delta(t-6.3)
$$




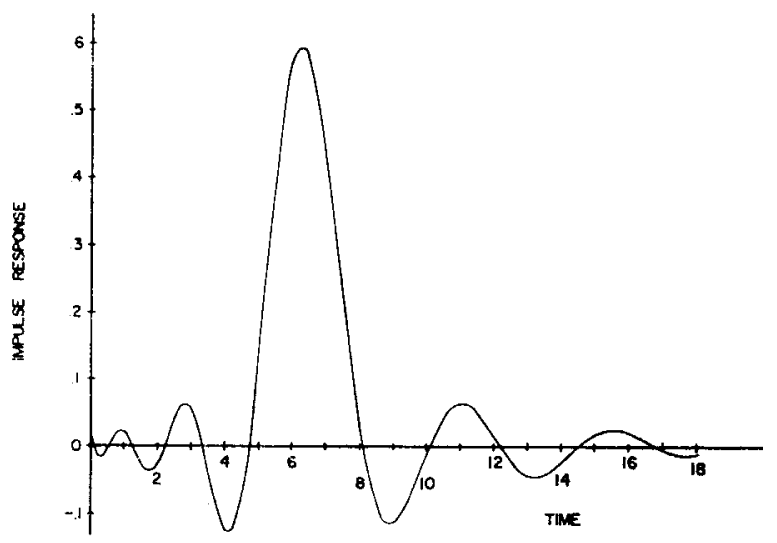

Fig. 1. Impulse response of eighth-order delay network.

is considered. This problem is classical in time-domain synthesis (13) and solution of it provides a severe test for any approximation technique, since, $f(t)$ is not in $L^{2}$. Sixteen Laguerre coefficients

$$
f_{n}=\lambda_{n}(6.3) \quad n=0, \cdots, 15
$$

were used, and an eighth-order model was chosen. The resulting approximation is

$$
\hat{F}(s)=\frac{0.02191 s^{7}+0.05325 s^{6}-2.013 s^{5}+11.93 s^{4}-35.32 s^{3}+59.84 s^{2}-56.20 s+23.04}{s^{8}+8.823 s^{7}+30.52 s^{6}+86.42 s^{5}+142.6 s^{4}+189.8 s^{3}+161.6 s^{2}+89.29 s+23.00} .
$$

Figures 1 and 2 show the impulse and step response of this delay network. The ratio of delay to 5-95 per cent rise-time of the step response is 3.5, which compares favorably with other time domain synthesis procedures for an eighthorder network (4).

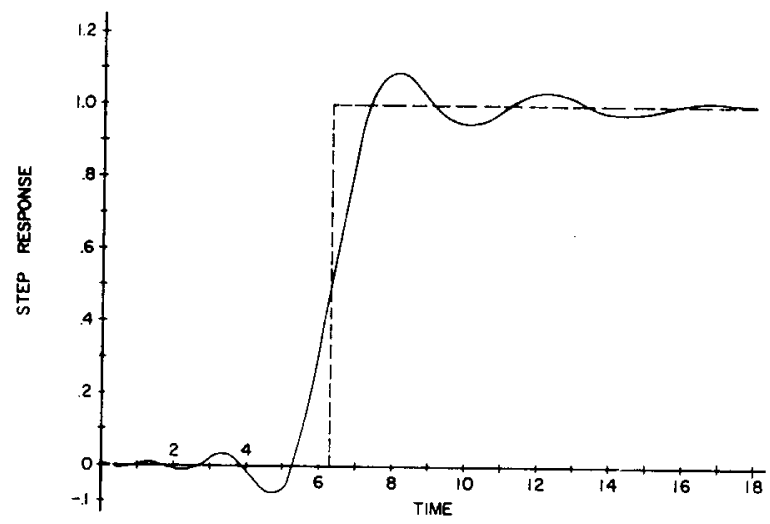

FIG. 2. Step response of eighth-order delay network. 


\section{Summary}

A method for the determination of an $n^{\text {th }}$ order rational transform for a time function, given at least $n+1$ of its Laguerre coefficients, has been presented with, as an illustration of the method, an application to a problem of lumped delay-line synthesis.

The method is advantageous in that: The poles are not predetermined, permitting $2 n$ degrees of freedom; the errors are under the control of the user; as many Laguerre coefficients as are available can be used, without increasing the complexity of the model; and the main computational effort involves only the inversion of an $n \times n$ matrix, easily done even for large $n$ on an automatic digital computer.

By using convolution techniques to measure the Laguerre coefficients of an unknown plant impulse response, the method can be used for tracking plant transfer functions in control applications.

\section{References}

(1) Elkind, Green, and Starr, "Application of Multiple Regression Analysis to Identification of Time-varying Linear Dynamic Systems," 1962 Joint Automatic Control Conference, Section 4-3, pp. 1-9, New York, New York.

(2) B. Liu, "Matching of Initial Behavior in Time Domain Approximation," Jour. Frank. Inst., Vol. 277, No. 2, pp. 107-118, Feb. 1964.

(3) M. Strieby, "A Fourier Method for Time Domain Synthesis," Proc. of Symposium on Modern Network Theory, Polytechnic Inst. of Brooklyn, pp. 197-209, Apr. 1955.

(4) B. Liu, "A Time Domain Approximation Method and Its Application to Lumped Delay Lines," IRE Trans. on Circuit Theory, Vol. CT-9, No. 3, pp. 256-61, Sept. 1962.

(5) G. C. Lendaris, "The Identification of Linear Systems," Trans. AIEE, Vol. 81 (Appl. and Ind.), pp. 231-242, Sept. 1962.

(6) M. J. Levin, "Estimation of a System Pulse Transfer Function in the Presence of Noise," IEEE Trans. on Automatic Control, Vol. AC-9, No. 3, pp. 229-235, July 1964.

(7) W. C. Yengst, "Approximation to a Specified Time Response," IRE Trans. on Circuit Theory, Vol. CT-9, No. 2, pp. 152-62; June 1962.

(8) F. B. Hildebrand, "Introduction to Numerical Analysis," New York, McGraw-Hill, pp. 378-379, 1956.

(9) L. Weiss, and R. N. McDonough, "Prony's Method, Z-Transforms, and Pade Approximation," SIAM Review, Vol. 5, No. 2, pp. 145-9, April 1963.

(10) R. N. McDonough, "Representation and Analysis of Signals; Part XV, Matched Exponents for the Representation of Signals," Johns Hopkins Univ., Dept. of Elec. Eng., April 30, 1963, report to Air Force Cambridge Research Center, Contract AF 19(604)1941.

(11) N. Wiener, "Extrapolation, Interpolation, and Smoothing of Stationary Time Series," New York, John Wiley and Sons, pp. 35-6, 1960.

(12) E. C. Titchmarsh, "The Theory of Functions," Oxford, England, Oxford University Press. Sec. 13.62, 1939.

(13) E. A. Guillemin, "Synthesis of Passive Networks," New York, John Wiley and Sons, pp. $690-707,1957$.

(14) R. E. Kalman, "Design of a Self-Optimizing Control System," Trans. ASME, Vol. 80, pp. 468-78, Feb. 1958. 